\title{
Massless Scalar Degrees of Freedom in QCD and in the Electroweak Sector from the Trace Anomaly ${ }^{1}$
}

\author{
Luigi Delle Rose and Mirko Serino \\ Dipartimento di Matematica e Fisica "Ennio De Giorgi" \\ Università del Salento \\ and \\ INFN Lecce, Via Arnesano 73100 Lecce, Italy
}

\begin{abstract}
The interaction of QCD and electroweak sector with gravity is characterized by the generation of a massless pole in a specific form factor present in the 1-loop effective action. We briefly illustrate how to single out this behaviour in perturbation theory, by taking as an example the $T J J$ vertex, with $T$ denoting the energy momentum tensor of the Standard Model. The breaking of scale invariance, due to the trace anomaly, is then related to the appearance of a local degree of freedom in the effective action, which is of dilaton type.
\end{abstract}

Keywords: Trace anomaly, perturbation theory, symmetry breaking, conformal symmetry

PACS: $11.25 . \mathrm{Db}, 11.25 . \mathrm{Hf}, 11.30 . \mathrm{Qc}$

\section{INTRODUCTION}

The insertion of the symmetric energy momentum tensor (EMT) on any generic correlator of a given theory defines the coupling of the same theory to gravity, describing the response of Green functions involving the theory currents to external gravitational perturbations. Mixed correlation functions involving one or more powers of the EMT and gauge currents play, in this respect, a special role, since they exhibit the breaking of scale symmetry. Such a breaking is induced by renormalization and is related to the appearance of a trace anomaly in these amplitudes.

An analysis of the effective action describing the interaction of a gauge theory with gravity shows that such an action is characterized by the appearance of an effective degree of freedom of dilaton type ([1]). This is seen as a massless pole in the structure of these Green functions at leading order in the gravitational coupling in the $T J J$ vertex, where $J$ denotes a neutral gauge current. We intend to summarize these findings, which result from a complex but quite direct analysis of these correlators, having been established for QED

\footnotetext{
${ }^{1}$ Presented at $Q C D @$ work, Lecce, June 18-21 2012
} 
([2, 3]), QCD [4] and for the complete electroweak sector [5].

\section{PERTURBATIVE EXPANSION}

We start with few definitions, focusing our discussions only on the case of the graviton/photon/photon $(T A A)$ vertex.

We recall that the fundamental action describing the coupling of gravity to the Standard Model is defined by the three contributions

$$
S=S_{G}+S_{S M}+S_{I}=-\frac{1}{\kappa^{2}} \int d^{4} x \sqrt{-g} R+\int d^{4} x \sqrt{-g} \mathscr{L}_{S M}+\frac{1}{6} \int d^{4} x \sqrt{-g} R \mathscr{H}^{\dagger} \mathscr{H},
$$

where $\kappa^{2}=16 \pi G_{N}$, with $G_{N}$ being the four dimensional Newton's constant and $\mathscr{H}$ is the Higgs doublet. We have denoted with $S_{G}$ the contribution from gravity (EinsteinHilbert term) while $S_{S M}$ is the Standard Model (SM) quantum action, extended to curved spacetime. $S_{I}$ denotes the term of improvement for the scalars, which are coupled to the metric via its scalar curvature $R$. The factor $1 / 6$ should be recognized as giving a conformally coupled Lorentz scalar, the $S U(2)$ Higgs doublet.

We denote with $T_{\mu \nu}$ the complete (quantum) EMT of the electroweak sector of the Standard Model. This includes the contributions of all the physical fields and of the Goldstones and ghosts in the broken electroweak phase. Its expression is uniquely given by the coupling of the Standard Model Lagrangian to gravity, modulo the terms of improvements, which depend on the choice of the coupling of the Higgs doublets. As we have mentioned, we have chosen a conformally coupled Higgs field. Our computation is performed in the $R_{\xi}$ gauge. The expression of the EMT is symmetric and conserved. It is therefore given by a minimal contribution $T_{M i n}^{\mu v}$ (without improvement) and the improvement EMT, $T_{I}^{\mu v}$, with

$$
T^{\mu v}=T_{M i n}^{\mu v}+T_{I}^{\mu v},
$$

where the minimal tensor is decomposed into

$$
T_{\text {Min }}^{\mu v}=T_{\text {f.s. }}^{\mu v}+T_{\text {ferm. }}^{\mu v}+T_{\text {Higgs }}^{\mu v}+T_{\text {Yukawa }}^{\mu v}+T_{\text {g.fix. }}^{\mu v}+T_{\text {ghost }}^{\mu v} .
$$

The various contributions refer, respectively, to the gauge kinetic terms (field strength, $f . s$.), the fermions, the Higgs, Yukawa, gauge fixing contributions ( $g$.fix.) and the contributions coming from the ghost sector.

\section{THE TAA CASE}

In the TAA case, we introduce the notation $\Gamma^{(A A) \mu v \alpha \beta}(p, q)$ to denote the one-loop amputated vertex function with a graviton and two on-shell photons. In momentum space we 
indicate with $k$ the momentum of the incoming graviton and with $p$ and $q$ the momenta of the two photons.

The amputated correlator is decomposed in the form

$$
\Gamma^{(A A) \mu v \alpha \beta}(p, q)=\Gamma_{F}^{(A A) \mu v \alpha \beta}(p, q)+\Gamma_{B}^{(A A) \mu v \alpha \beta}(p, q)+\Gamma_{I}^{(A A) \mu v \alpha \beta}(p, q),
$$

as a sum of a fermion sector (F), a gauge boson sector (B) and a term of improvement denoted as $\Gamma_{I}^{\mu v \alpha \beta}$. A complete computation gives for the various gauge invariant subsectors the explicit expressions

$$
\begin{aligned}
& \Gamma_{F}^{(A A) \mu v \alpha \beta}(p, q)=\sum_{i=1}^{3} \Phi_{i F}\left(s, 0,0, m_{f}^{2}\right) \phi_{i}^{\mu v \alpha \beta}(p, q) \\
& \Gamma_{B}^{(A A) \mu v \alpha \beta}(p, q)=\sum_{i=1}^{3} \Phi_{i B}\left(s, 0,0, M_{W}^{2}\right) \phi_{i}^{\mu v \alpha \beta}(p, q) \\
& \Gamma_{I}^{(A A) \mu v \alpha \beta}(p, q)=\Phi_{1 I}\left(s, 0,0, M_{W}^{2}\right) \phi_{1}^{\mu v \alpha \beta}(p, q)+\Phi_{4 I}\left(s, 0,0, M_{W}^{2}\right) \phi_{4}^{\mu v \alpha \beta}(p, q)
\end{aligned}
$$

in terms of form factors $\Phi_{i}$ 's (see [5] for all of these and the $\phi_{i}^{\mu v \alpha \beta}(p, q)$ 's). The first three arguments of the form factors stand for the three independent kinematical invariants $k^{2}=(p+q)^{2}=s, p^{2}=q^{2}=0$ while the remaining ones denote the particle masses circulating in the loop. We have used the on-shell renormalization scheme. In the TAA vertex, the contribution to the trace anomaly in the fermion sector comes from $\Phi_{1 F}$ which is given by

$$
\begin{aligned}
\Phi_{1 F}\left(s, 0,0, m_{f}^{2}\right)= & -i \frac{\kappa}{2} \frac{\alpha}{3 \pi s} \sum_{f} Q_{f}^{2}\left\{-\frac{2}{3}+\frac{4 m_{f}^{2}}{s}\right. \\
& \left.-2 m_{f}^{2} C_{0}\left(s, 0,0, m_{f}^{2}, m_{f}^{2}, m_{f}^{2}\right)\left[1-\frac{4 m_{f}^{2}}{s}\right]\right\},
\end{aligned}
$$

with the sum taken over all the fermions $(f)$ of the Standard Model. $C_{0}$ denotes the threepoint scalar integral (see [5]) and $m_{i}$ are the fermion masses. As one can immediately realize, this form factor is characterized by the presence of an anomaly pole

$$
\Phi_{1 p o l e}^{F} \equiv i \kappa \frac{\alpha}{9 \pi s} \sum_{f} Q_{f}^{2}
$$

which is responsible for the generation of the anomaly in the massless limit. This pattern is typical of any gauge invariant correlator of the Standard Model. The other gauge-invariant sector of the $T A A$ vertex, in the electroweak case, is the one mediated by the exchange of 
bosons, Goldstones and ghosts in the loop. We will denote with $M_{W}$ the mass of the W's. In this sector the form factor contributing to the trace is

$$
\begin{aligned}
\Phi_{1 B}\left(s, 0,0, M_{W}^{2}\right)= & -i \frac{\kappa}{2} \frac{\alpha}{\pi s}\left\{\frac{5}{6}-\frac{2 M_{W}^{2}}{s}\right. \\
& \left.+2 M_{W}^{2} C_{0}\left(s, 0,0, M_{W}^{2}, M_{W}^{2}, M_{W}^{2}\right)\left[1-\frac{2 M_{W}^{2}}{s}\right]\right\},
\end{aligned}
$$

which multiplies the tensor structure $\phi_{1}$, responsible for the generation of the anomalous trace. In this case the anomaly pole is easily isolated from (10) in the form

$$
\Phi_{1 B, p o l e} \equiv-i \frac{\kappa}{2} \frac{\alpha}{\pi s} \frac{5}{6} .
$$

The term of improvement is responsible for the generation of two form factors, both of them contributing to the trace. They are given by

$$
\begin{aligned}
& \Phi_{1 I}\left(s, 0,0, M_{W}^{2}\right)=-i \frac{\kappa}{2} \frac{\alpha}{3 \pi s}\left\{1+2 M_{W}^{2} C_{0}\left(s, 0,0, M_{W}^{2}, M_{W}^{2}, M_{W}^{2}\right)\right\} \\
& \Phi_{4 I}\left(s, 0,0, M_{W}^{2}\right)=i \frac{\kappa}{2} \frac{\alpha}{6 \pi} M_{W}^{2} C_{0}\left(s, 0,0, M_{W}^{2}, M_{W}^{2}, M_{W}^{2}\right)
\end{aligned}
$$

the first of them being characterized by an anomaly pole

$$
\Phi_{1 I \text { pole }}=-i \frac{\kappa}{2} \frac{\alpha}{3 \pi s} .
$$

The same situation is encountered in QCD, when $J$ denotes a gluon current: here both the gluon loop and the fermion loop are separately gauge invariant and both exhibit such a structure.

\section{IMPLICATIONS FOR THE EFFECTIVE ACTION}

The pole-like behaviour generated by the anomaly diagrams have a general implication concerning the structure of the effective action. We recall [2, 4] that in the case of massless QED the effective interaction induced by the trace anomaly takes the form

$$
\mathscr{S} \sim \int d^{4} x d^{4} y R^{(1)}(x) \square^{-1}(x, y) F_{\mu v}(y) F^{\mu v}(y)
$$

where $R^{(1)}$ denotes the linearized scalar curvature and $F_{\mu \nu}$ is the abelian field strength. A similar result holds for QCD [4]. As shown in [2] this expression coincides with the 
long-known anomaly-induced action obtained by Riegert [6], which was derived for a generic gravitational field, after an expansion of its expression around the flat spacetime limit. Notice that in terms of auxiliary degrees of freedom (i.e. two scalar fields $\left(\varphi, \psi^{\prime}\right)$ ) which render the action (15) local [2], extra couplings of the form $\varphi F F$ are automatically induced by the $1 / \square$ term. This interaction is indeed present in the equivalent Lagrangian

$$
S_{\text {anom }}\left[g, A ; \varphi, \psi^{\prime}\right]=\int d^{4} x \sqrt{-g}\left[-\psi^{\prime} \square \varphi-\frac{R}{3} \psi^{\prime}+\frac{c}{2} F_{\alpha \beta} F^{\alpha \beta} \varphi\right],
$$

$(c=-\beta(e) /(2 e))$ where $\varphi$ and $\psi^{\prime}$ are auxiliary scalar fields. $\varphi$ describes a dilaton interaction.

\section{CONCLUSIONS}

These findings seem to indicate that the signature of the trace anomaly is in the appearance of a massless degree of freedom of dilaton type which force us to draw a parallel between this anomaly and the chiral anomaly. In the chiral case, anomaly poles have been a distinctive signature of the anomaly for almost two decades, which in a local formulation of the effective Lagrangian amount to axion-like interactions, in the form of Wess-Zumino terms [7], studied in the context of anomalous $U(1)$ extensions of the Standard Model [8, 9]. There are several implications of these findings. We have discussed in a related contribution to these proceedings the role of such dilaton-like degree of freedom in the context of conformal extensions of the Standard Model, and its possible appearance, in the broken electroweak phase, together with the Higgs scalar [1]. There are also other implications. We mention, for instance, the case of supersymmetric theories, where the anomaly multiplet is known to contain as components the trace of the EMT, an axial anomaly related to the $U(1)_{R}$ current $\left(J_{R}\right)$ and the gamma-trace of the supersymmetric current. Our results clearly imply that such poles are present when we insert two (i.e. $T$ and $J_{R}$ ) of these three operators in the effective action. Threfore they should also appear in correlators involving insertions of the supersymmetric current.

\section{REFERENCES}

1. C. Corianò, L. Delle Rose, A. Quintavalle and M. Serino (2012), 1206.0590.

2. M. Giannotti, and E. Mottola, Phys. Rev. D79, 045014 (2009), 0812 . 0351.

3. R. Armillis, C. Corianò, and L. Delle Rose (2010), Phys. Rev. D81, 085001 (2010), 0812.0351

4. R. Armillis, C. Corianò, and L. Delle Rose (2010), Phys.Rev. D82, 064023 (2010), 1005.4173.

5. C. Corianò, L. Delle Rose, and M. Serino, Phys.Rev. D83, 125028 (2011), 1102.4558.

6. R. J. Riegert, Phys. Lett. B134, 56-60 (1984).

7. R. Armillis, C. Corianò, and L. Delle Rose, Phys. Lett. B682, 322-327 (2009), 0909.4522

8. C. Corianò, N. Irges, and E. Kiritsis, Nucl. Phys. B746, 77-135 (2006), hep-ph/ 0510332.

9. C. Corianò, N. Irges, and S. Morelli, Nucl. Phys. B789, 133-174 (2008), hep-ph/0 03127. 